This document is the accepted manuscript version of the following article: Horváth, B., A1 Jassin-A1-Hashemi, E., \& Schift, H. (2019). Interdigitated electrode arrays made by capillary filling and spin coating for UV-sensor fabrication. Flexible and Printed Electronics, 4(3), 035002 (10 pp.). https://doi .org/10.1088/2058-8585/ab3777

\title{
Interdigitated electrode arrays made by capillary filling and spin coating for UV-sensor fabrication
}

\author{
Barbara Horváth ${ }^{1}$, Ethouba Al Jassin-Al-Hashemi ${ }^{1,2}$, and Helmut Schift ${ }^{{ }^{*}}$ \\ ${ }^{1}$ Paul Scherrer Institute (PSI), Laboratory for Micro- and Nanotechnology, 5232 Villigen PSI, \\ Switzerland \\ ${ }^{2}$ University of Basel, Department of Physics, Klingelbergstr. 80, 4056 Basel, Switzerland \\ *Corresponding author: Helmut Schift, e-mail: helmut.schift@psi.ch
}

Keywords: UV-sensor, nanoparticle ink, self-confinement, nanoimprint lithography, interdigitated electrode array

\begin{abstract}
In this paper the fabrication of UV-sensors based on an interdigitated electrode array with a 70 $\mathrm{nm}$ sputtered $\mathrm{ZnO}$ coating is demonstrated. The electrodes were fabricated using polymer substrates prepatterned with nanoimprint lithography, imprinted with $\mu \mathrm{m}$-sized grooves and reservoirs at two sides. The V-shaped capillaries were filled with nanoparticle based silver ink either by capillary action in an open-fluidics approach or with spin coating. This enables the use of low-resolution additive methods to fabricate high precision wires for low cost flexible electronics. The $1 \times 1 \mathrm{~mm}^{2}$ sensor area contained 100 wires in alternating directions that were electrically insulated from each other, and were attached to contact pads. Characterization of UV sensor devices was carried out experimentally by performing electrical measurements under 355 and $385 \mathrm{~nm}$ UV light illumination.
\end{abstract}

\section{Introduction}

Inkjet and gravure printing are low-cost additive methods used in flexible electronics with resolutions mostly in the range of over $20 \mu \mathrm{m}$ for inkjet and $5 \mu \mathrm{m}$ for gravure printing [14]. Individual droplets of ink are deposited on the surface by jetting or by mechanical transfer from cells on a printing cylinder to a substrate. Conducting electrical wires are generated by connecting the silver nanoparticle-based ink droplets that are containing nanoparticles dispersed in a suitable solvent. Ink volumes of a few picoliters (or even femtoliters in case of gravure printing) are deposited on the substrate on which the ink is able to spread, and then further processed into electrically conducting wires by drying and sintering. By pre-patterning the substrate with $\mu \mathrm{m}$-sized grooves by nanoimprint lithography (NIL), inks can be deposited on the surface of the patterns or inside the grooves in an open-fluidics approach [5-10].

An open-fluidics capillary method has been applied to the fabrication of top-gate thinfilm transistors on plastic by using the concept of self-aligned capillarity-assisted lithography in electronics (SCALE) [11]. The dimensions of the channels ranged from a few micrometers to tens of micrometers and they are connected to larger reservoirs. The use of multi-tier 
structures enabled control of flow (e.g. for creating stoppers and diodes) and the subsequent filling with different materials for fabrication of resistors and transistors [12-14]. For the smaller channels, which are at the bottom level, a reactive (particle-free) Ag ink was used. This enabled to conformally coat the channel walls with a thin layer of silver, which kept the channel open for subsequent filling steps. Typically, the ink droplet deposited in the reservoir is much larger than the grooves and spreading can be controlled by surface topography [15]. However, significant evaporation along channels has to be taken into account while filling long micrometer-sized grooves, which leads to changes in viscosity over the spreading distance. In previous research, using nanoparticle-based inks, we have explored to which length capillaries can be filled and why the final thickness of the metallic wires is higher than expected by the solid content in the nanoparticle dispersion [16]. The main reason is that for the capillary widths of 2 to $5 \mu \mathrm{m}$ an ongoing filling occurs from the reservoir during evaporation of the solvent. According to [17], the evaporation at the open surface of the liquid inside the channel induces a convective flow of ink from the reservoir, until the particles concentrate to form a densely packed metallic deposit starting at the end of the channel and propagating all the way back to the reservoir. This assures that more ink particles are transported into the capillaries, which upon drying concentrate in the capillaries before they are further densified and converted into conductive wires. In contrast to this, the line length seems to be limited and the ink is not continuing to flow as it would be predicted by capillary flow theory [18-21]. This way, a $2 \mu \mathrm{m}$ deep, $500 \mu \mathrm{m}$ long U-groove can be used to generate an $1 \mu \mathrm{m}$ thick metallic wire, by using an ink dispersion with only $1.4 \mathrm{v} / \mathrm{v} \%$ of particles. The shape of the channel has an effect on the travel speed and length: V-shaped capillaries (V-grooves) spread slower and shorter distances than U-shaped capillaries of the same width [16].

Spin coating is an alternative way to place nanoparticles into the trenches. As described in our previous research [22], nanoparticles can deposit in the bottom of V-grooves with inclined sidewalls after spin coating heavily diluted Ag inks in alcohol. The rotation during spin coating removes excess material from above the grooves. The nanoparticles then merge into an agglomerated line on the bottom of the vertex of the $\mathrm{V}$-grooves after evaporation of the solvent, and a following sintering step removes the organic additives forming a metal wire. By using Vgrooves, the spin coating process allows the generation of sub-micron wide wires (down to $\sim 100 \mathrm{~nm}$ range) which are significantly smaller than the width of the V-grooves that were produced by using pre-patterned substrates.

In the research presented here, we apply the capillary and spin coating method to fabricate a UV-sensor, which is a large area interdigitated electrode array (IDEA) covered with a semiconductor sensitive to UV-light [23]. For capillary filling, the main challenge is that many parallel capillaries have to be filled with a total volume comparable to the inking reservoir volume serving as electrical contact. Furthermore, the fabrication of dense arrays of finger-like electrodes for a device requires high quality of electrodes: defects such as spill-over lead to shortcuts between alternating electrodes and therefore have to be completely avoided, breaks within single electrodes however only lead to a local reduction in sensitivity. The spin coating method enables to create wires within $\mathrm{V}$-grooves by self-confinement while the density in nonpatterned areas is too low to form a conductive layer [22]. For generation of the UV-sensitive layer a standard sputtering method for the zinc oxide $(\mathrm{ZnO})$ on the surface of the IDEAs was used instead of method compatible with low cost fabrication (e.g. by ink jet printing on top of the IDEA). The findings of our research will help to scale up the technique to larger areas, 
define rules for capillary filling of the inks, and reduce the effect of this approach on postprocessing.

\section{Experimental}

Figure 1 shows the fabrication steps for creating $\mathrm{V}$-groove patterning on plastic substrates. For the fabrication of the stamps, <100> Si wafers (diameter $100 \mathrm{~mm}$ ) were coated with a $100 \mathrm{~nm} \mathrm{Si}{ }_{3} \mathrm{~N}_{4}$ film by LPCVD. On this substrate a negative resist (AZ nLOF 2020, MicroChem) was spin coated and prebaked at $110^{\circ} \mathrm{C}$ for $90 \mathrm{~s}$. Contact photolithography was applied to pattern the resist (design width $5 \mu \mathrm{m}$ ), which was then postbaked at $110^{\circ} \mathrm{C}$ for $120 \mathrm{~s}$. After developing the resist, etching of the $\mathrm{Si}_{3} \mathrm{~N}_{4}$ was done by RIE in a mixture of $\mathrm{O}_{2} 5 \mathrm{sccm}$, and $\mathrm{CHF}_{3} 40 \mathrm{sccm}$, at a $100 \mathrm{~nm} / \mathrm{min}$ etching rate. Potassium hydroxide $(\mathrm{KOH})$-etching was applied on the exposed $\mathrm{Si}$ at $80^{\circ} \mathrm{C}$ for $3 \mathrm{~min}$ and the $\mathrm{Si}_{3} \mathrm{~N}_{4}$ masking layer was then removed by RIE. V-groove lines with an angle of $54.74^{\circ}$ between the $\langle 100\rangle$ and the $\langle 111\rangle$ crystalline planes were formed in the silicon wafer surface. The substrate with V-grooves was inverted into a stamp with $\Lambda$-ridges: with $U V$-assisted imprint, Ormostamp (micro resist technology) was spin coated on the substrate that solidified after UV irradiation, and this stamp was then detached from the Si substrate. The stamps were thermally imprinted (Jenoptik HEX03) into a $175 \mu \mathrm{m}$ thick poly (methyl methacrylate) (PMMA, glass transition temperature $\mathrm{T}_{\mathrm{g}}=113^{\circ} \mathrm{C}$ ) film for $10 \mathrm{~min}, 20 \mathrm{kN}$ at $180^{\circ} \mathrm{C}$.

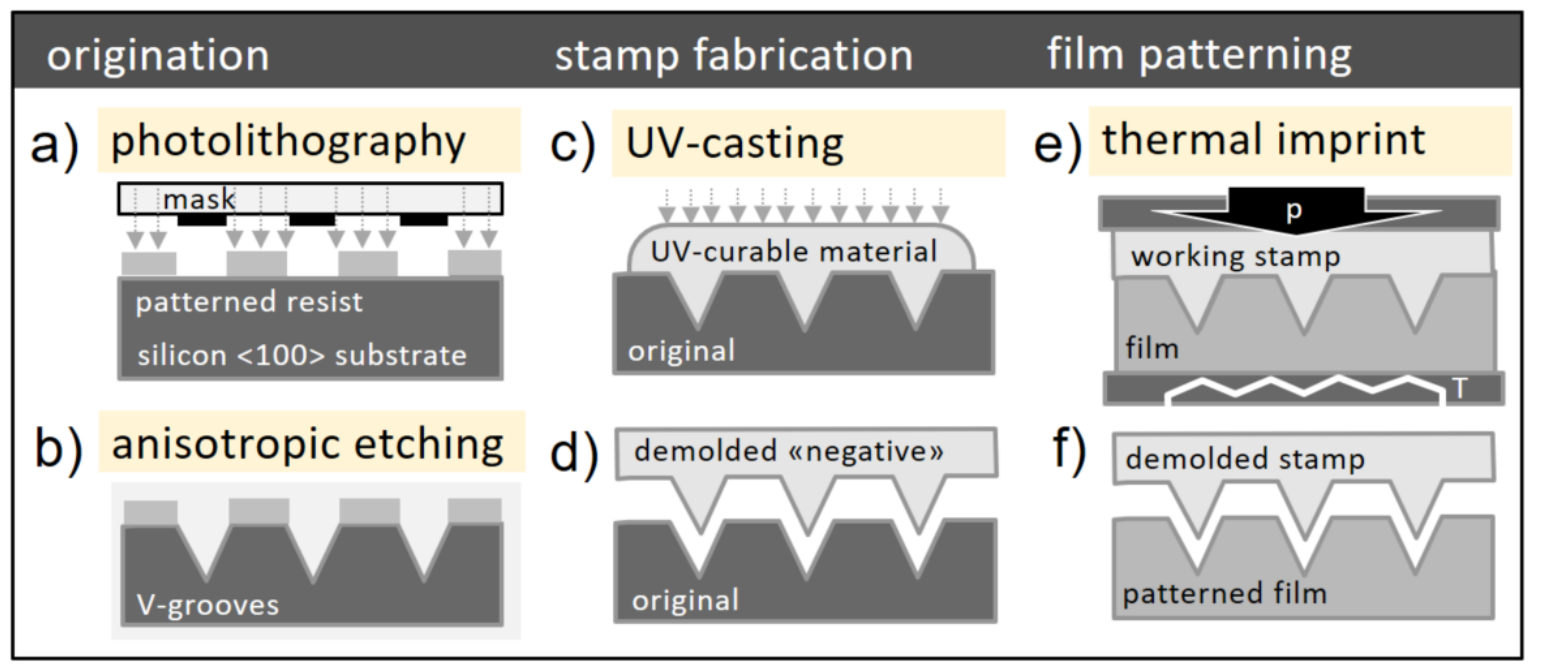

Figure 1. Patterning of V-grooves into polymeric films by generation of an original using a) mask-based photolithography and b) wet anisotropic etching; c, d) generation of a working stamp "negative" with inverted V-grooves using UV-casting; and e, f) replication of V-groove structures into polymer films by thermal NIL.

Three different designs of IDEAs were created: the etched V-grooves were always 5.4 $\mu \mathrm{m}$ wide and $3.8 \mu \mathrm{m}$ deep, the distance between two electrodes were $4.6 \mu \mathrm{m}, 9.6 \mu \mathrm{m}$ and 14.6 $\mu \mathrm{m}$, here denoted with $(5 / 10),(5 / 15)$ and $(5 / 20)$ according to their design, where the first number indicates line width and the second number indicates the period. These patterned PMMA films were covered with Ag nanoparticle ink dispersions either by capillary filling or by spin coating. For capillary filling, Smart'Ink S-CS01130 (Genes'Ink) in a carrier vehicle of alcohol and alkane with a specified nanoparticle loading of $20 \mathrm{w} / \mathrm{w} \%, \sim 1.4 \mathrm{v} / \mathrm{v} \%$ concentration, 
$\sim 13 \mathrm{mPa} \cdot \mathrm{s}$ viscosity (given in the supplier's data sheets) was used. The contact angle of Smart'Ink S-CS01130 on PMMA is $\theta=6^{\circ}$. The Ag inks were deposited using a Biospot BT600 plotter from BioFluidiX. The volume of the droplets were $5 \mathrm{~nL}$ (corresponds to a spherical droplet of $210 \mu \mathrm{m}$ diameter, fitting well for the $500 \times 500 \mu \mathrm{m}^{2}$ reservoirs). Spin coating was done with a dilution of Smart'Ink S-CS31506 (Genes'Ink) in a carrier vehicle of alcohol and glycol (nanoparticle loading of 55+/-5 w/w\%, 150-400 mPa.s viscosity), further thinned down with isopropyl alcohol (IPA) in a volume ratio of 1:59. This $1: 59$ dilution has $0.17 \mathrm{v} / \mathrm{v} \%$ concentration and a viscosity close to IPA $\left(2.4 \mathrm{mPa} \cdot \mathrm{s}\right.$ at $20^{\circ} \mathrm{C}$ without nanoparticles). This solution was spin coated on the patterned samples for $120 \mathrm{~s}$ at $5000 \mathrm{rpm}$. An additional droplet of S-CS31506 ink has been placed on the filled reservoirs of both spin coated and capillary samples just before sintering - this viscous ink did not flow into the capillaries, its role is solely to ensure a better electrical connection of the contact pad to the probes for electrical measurements. Sintering of the $\mathrm{Ag}$ wires has been performed on a hotplate immediately after for $30 \mathrm{~min}$ at $150{ }^{\circ} \mathrm{C}$ for capillary filling and $180{ }^{\circ} \mathrm{C}$ for spin coated inks. A $70 \mathrm{~nm}$ thick zinc oxide $(\mathrm{ZnO})$ layer has been sputtered on top of the electrodes with no masking layer using a sputter coater (CT200 sputter cluster, Alliance concept) with: pressure 4.10-3 mbar, Ar $60 \mathrm{sccm}$, RF power $100 \mathrm{~W}$ for a $100 \mathrm{~mm} \mathrm{ZnO}$ target, deposition rate $2.5 \mathrm{~nm} / \mathrm{min}$. Samples were examined with an optical microscope (Leica INM 20) and a scanning electron microscope (SEM) (Zeiss Supra VP55) at an acceleration voltage of $0.5-1.0 \mathrm{kV}$.

Characterization of UV sensor devices was carried out experimentally by performing electrical measurements under UV light illumination. Resistivity measurements were performed by measuring the current flow after connecting the sample with a voltage source of 1-5 V. The IDEA was contacted by using manual probe heads from SUSS with steel probe tips and breaking through the $\mathrm{ZnO}$ layer on top of the contact pads. As UV-source, an unmounted LED was used with $355 \mathrm{~nm}$ (Lumex SSL- LXTO46355C) and $385 \mathrm{~nm}$ (Thorlabs M385F1) wavelength, and with an intensity of 0.080 and $0.087 \mathrm{~mW} / \mathrm{cm}^{2}$, respectively, at the distance of $10 \mathrm{~mm}$.

\section{Results and discussion}

\subsection{Filling of interdigitated electrodes with nanoparticle inks}

The strategy in this research is to pre-pattern a polymer substrate with $\mu \mathrm{m}$-sized grooves, and deposit ink into the grooves with established concepts, i.e. using capillary filling from the side along the grooves [16] or by spin coating from the top [22]. The surface topographies of the substrate consist of open wells and channels with slanted sidewalls that narrow down to the bottom of the channel. After nanoparticle deposition and drying, the Ag particle-based inks (typically with 20 to $80 \mathrm{~nm}$ nanoparticles dispersed in a suitable solvent) need to be sintered [24]. The nanoparticles merge into a densely packed agglomerated line after drying. Sintering removes the organic additives surrounding the nanoparticles and a continuous metal wire is formed by the diffusion of $\mathrm{Ag}$ atoms with conductivity close to bulk metal.

\section{Capillary filling}

During capillary filling the sidewalls of the channels exert high pulling forces on the viscous liquid which drags them along the capillaries due to Laplace pressure and surface tension [25]. Such open microfluidics becomes attractive because of its easy manufacturing and 
accessibility and is particularly suitable for nanoparticle inks. The aim is that most of the nanoparticles are confined within the grooves, the ink does not wet the surrounding flat areas (plateaus) or spill over to neighboring grooves, and metal wires form only within the grooves upon sintering.

The concept is the following: multiple parallel interdigitated capillary arrays are connected to a reservoir from where the ink flow will start (Figure 1a) [26, 27]. In order to achieve a homogeneous wetting to the ink receiving area, we designed it with an array of parallel microcapillaries to help confining and spreading the dispensed ink droplet (Figure 2a). After dropping an ink droplet on the reservoir, the ink spreads to its boundaries, then penetrates into the capillaries and fills it up to a predefined distance. The filling of the electrode capillaries starts immediately when the ink reaches the capillaries. When designing IDEAs, the ink should reach the end of the electrodes, which makes the process reproducible. Wider capillaries carry the ink faster and the effects of evaporation are reduced due to the larger liquid volume. The droplet placement accuracy does not have a strong influence on the filling; as soon as a small portion of the droplet touches the reservoir, the ink will be directed to the trenches due to the design of the reservoir with guiding lines.

Figure $2 \mathrm{~b}$ shows an IDEA filled from both sides with large ink drops. The interdigitated area is $1000 \times 1000 \mu \mathrm{m}^{2}$ and the capillaries were attached to $500 \times 1000 \mu \mathrm{m}^{2}$ reservoirs that were structured with the same line shape, width and spacing as the attached capillaries. For $10 \mu \mathrm{m}$ period of wires (5/10 case), the 50 capillaries on each side are $1200 \mu \mathrm{m}$ long (within this a 1000 $\mu \mathrm{m}$ section is parallel to the opposite direction electrodes). An ink droplet of $\sim 5 \mathrm{~nL}$ is dropped on each reservoir, which spreads and fills the capillaries of the future contact pad and the electrodes. Occasionally, the filling is not entirely successful; as seen on Figure 2b, where a small area is not filled on the lower part (approx. 5 capillaries) (bright stripe). The entire volume of the V-groove capillaries in the designed IDEA is calculated to be $2.28 \mathrm{~nL}(2 \times 0.53 \mathrm{~nL}$ for each reservoir and $1.22 \mathrm{~nL}$ for the electrode arrays) for the (5/10) case. Therefore, if we apply $\sim 5 \mathrm{~nL}$ droplets on each reservoir, overfilling leads to a continuous ink supply to the electrode capillaries without depleting the reservoirs. This enables higher thickness (Figure 4a) due to the continuous transport of nanoparticles along capillaries during evaporation of the solvent [16]. Taking into account the dynamics of filling and evaporation (e.g., filling of capillaries while the ink droplet is not yet completely spread in the reservoir), inhomogeneous filling of capillaries could result. However, as seen in Figure 2b, most of the capillaries were filled up to the end. This goes along with our observations in [16] that the filling length is mostly defined by the viscosity and surface energy rather than by the amount of liquid present at the entrance of the capillary. It has to be noted that the filling heights are only rough estimates because the entire filling is happening within seconds and we have to take into account the formation of a meniscus of liquids in the capillaries. Since the capillaries have an end, the length of the electrodes is well defined. All calculations were done by anticipating a concave meniscus, which would result in even smaller values. 
a)

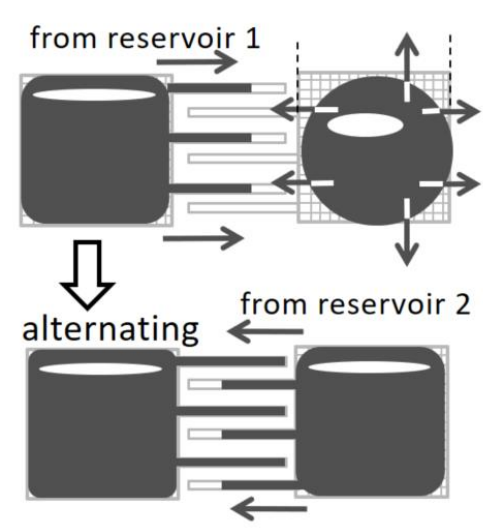

b)

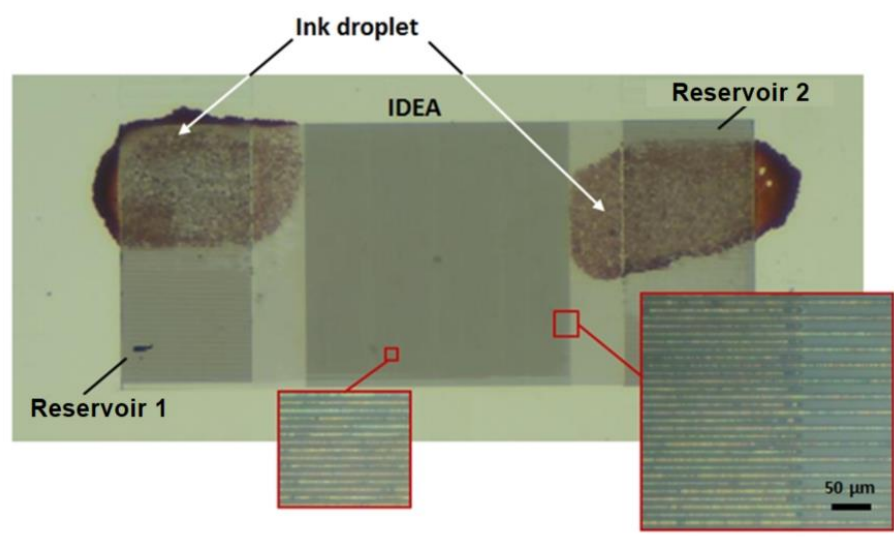

Figure 2. a) Concept of droplet deposition on open capillaries between two reservoirs. The schematic shows the filling sequence a) inking of the left reservoir and capillary filling, and inking of the right reservoir, thus creation of an array of interdigitated electrodes. b) Optical micrograph of a $1000 \times 1000 \mu \mathrm{m}^{2}$ IDEA with $1.2 \mathrm{~mm}$ long electrodes attached to contact pads.

\section{Spin coating}

Spin coating of highly diluted ink on the IDEA structures results in a complete coverage of the surface with ink particles (Figure 3a). It causes the substrate film to change its color to a silverish shade (Figure 3b), due to the low viscosity of the diluted ink and the low density of nanoparticles. Despite the coloring, a conducting film with connected nanoparticles does not form in the non-patterned regions of the sample, only independent nanoparticles are placed on the surface. The V-grooves, however, are completely filled with the ink dilution with a concave meniscus. This leads to the deposition of nanoparticles in the bottom of the V-groove after complete evaporation of the solvent. Since the sidewalls dewet during this evaporation, the resulting confined nanoparticle line will shrink not only vertically but also laterally. The diluted high viscosity ink results in a much lower nanoparticle volume content of about $0.17 \mathrm{v} / \mathrm{v} \%$ for the 1:59 ratio dilution. In this case, contact angle of the ink on the PMMA polymer is almost $0^{\circ}$. For $\mathrm{V}$-grooves with $3.2 \mu \mathrm{m}$ width and $2.3 \mu \mathrm{m}$ depth the resulting wire width was experimentally determined to be $630 \mathrm{~nm}$ before and $470 \mathrm{~nm}$ after sintering [22].
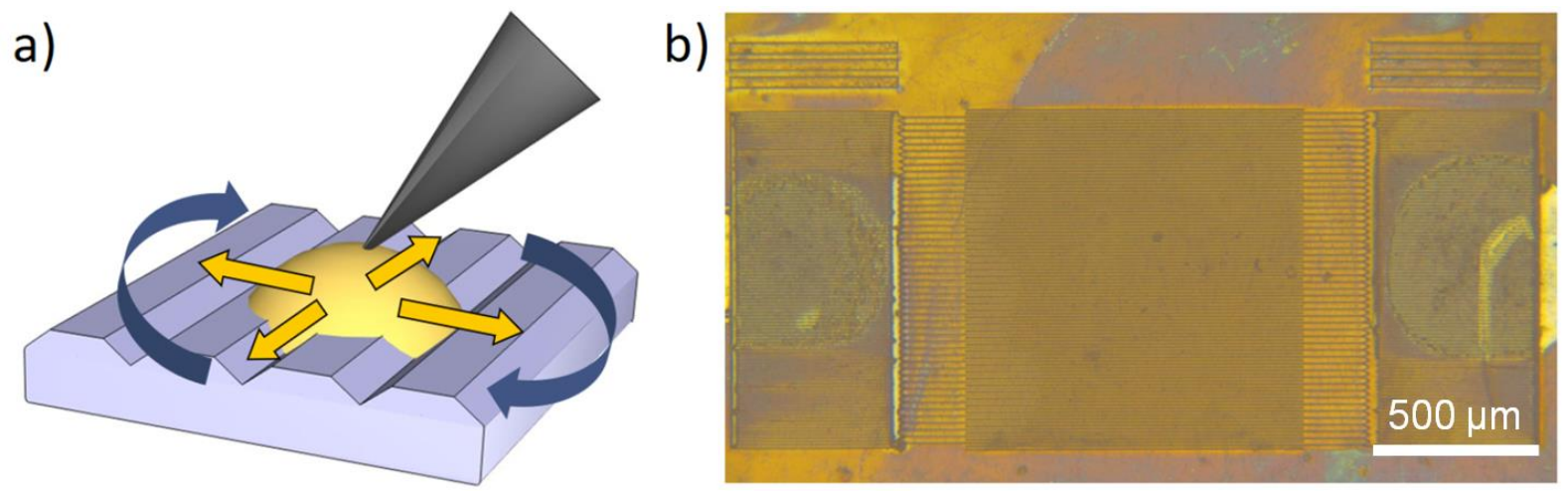

Figure 3. a) Concept of spin coating on open capillaries of IDEAs. b) Optical micrograph of a $1000 \times 1000 \mu \mathrm{m}^{2}$ IDEA after spin coating and $\mathrm{ZnO}$ deposition (the large droplets on the reservoirs are from undiluted ink not able to penetrate in the capillaries needed for electrical contact). 
A simple calculation of the number of $50 \mathrm{~nm}$ diameter particles shows that if a $10 \times 10 \mu \mathrm{m}^{2}$ area were covered with a $1-\mu \mathrm{m}$ thick $0.17 \mathrm{v} / \mathrm{v} \%$ nanoparticle dilution, then $\sim 2$ ' 600 particles would remain on the surface after complete evaporation of the solvent. This volume is identical to that of a $5.4 \mu \mathrm{m}$ wide, $3.8 \mu \mathrm{m}$ deep and $10 \mu \mathrm{m}$ long V-groove. Therefore, 4'000 particles would end up in a V-groove by sedimentation, while 1'200 particles covering the $4.6 \times 10 \mu \mathrm{m}^{2}$ plateau besides the $\mathrm{V}$-grooves would have $5 \%$ surface coverage, which is too sparse to form a connected and thus electrically conducting coating. In contrast to this, upon accumulation in the vertex of the $\mathrm{V}$-groove, the $0.17 \mathrm{v} / \mathrm{v} \%$ would result in a wire width of (1)

$\mathrm{W}_{\text {wire }}=\mathrm{WV}$-groove $\times 0.0017^{-1 / 3}$

if we anticipate a concentration in all three directions. This would result in $650 \mathrm{~nm}$ width without and $750 \mathrm{~nm}$ width with an additional $1-\mu \mathrm{m}$ thick coverage, respectively. This value is even larger if several other factors are anticipated, such as the inclusion of voids due to the densest sphere packing of $74 \%(710 / 820 \mathrm{~nm})$ and the formation of a slight meniscus in the center of the wire. SEM micrographs of surfaces show about $100-500$ particles in a $10 \times 10 \mu \mathrm{m}^{2}$ area, which is much less than the calculated 2'600 particles occurring from the $1-\mu \mathrm{m}$ thick coverage. However, we know that particles coalesce and even grow at low temperatures at the expense of smaller particles [22]. Therefore, we assume that the overall coverage is much thinner or that most particles outside the grooves are washed away with the solvent during spinning before the first particles stick to the surface. The simple calculation shows that at the 1:59 dilution chosen for this example coverage by spin coating within the V-grooves is high enough that conductive wires with a significant width can be generated, while the particles on the plateaus would not have any electrical functionality. Spin coating is a process preferred for solid, wafer-like substrates, but not easily scalable. For the homogeneous coating of large area polymer films, as needed for flexible electronics, reverse gravure printing would be a more appropriate process, requiring a viscosity in the range of $\sim 13 \mathrm{mPa} \cdot \mathrm{s}$ such as the undiluted Smart'Ink S-CS31506.

Comparison of wires made by capillary filling and spin coating 
a) Capillary filling from reservoir

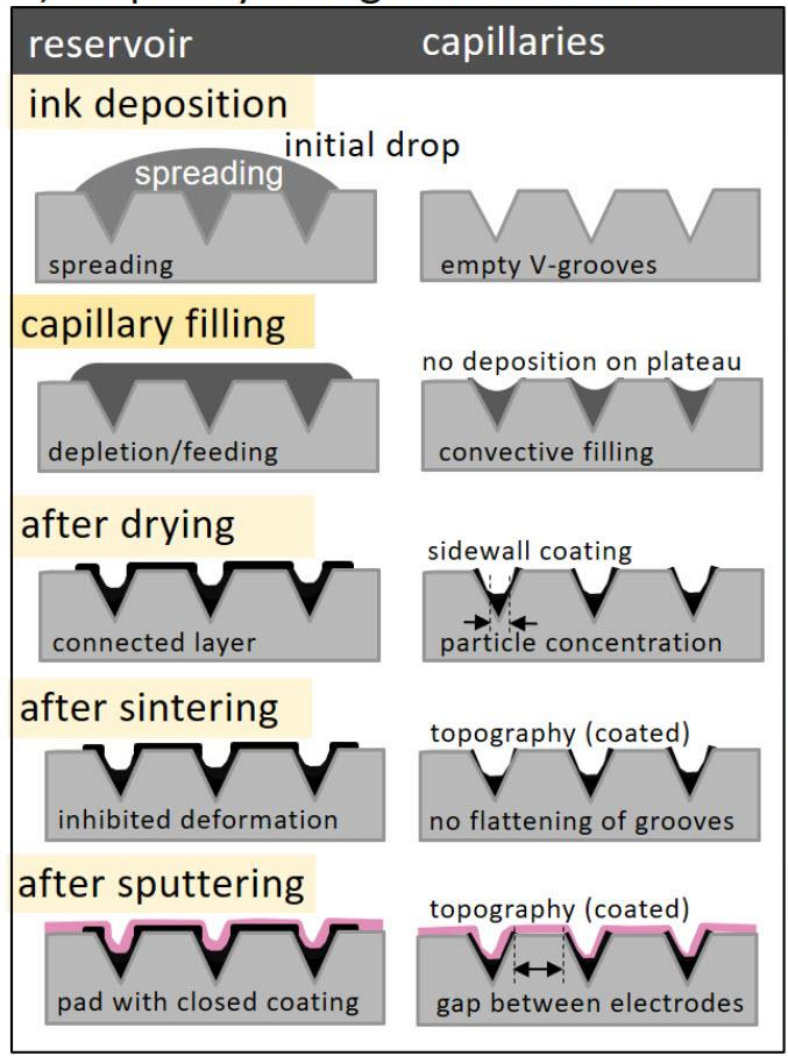

b) Spincoating and self-confinement

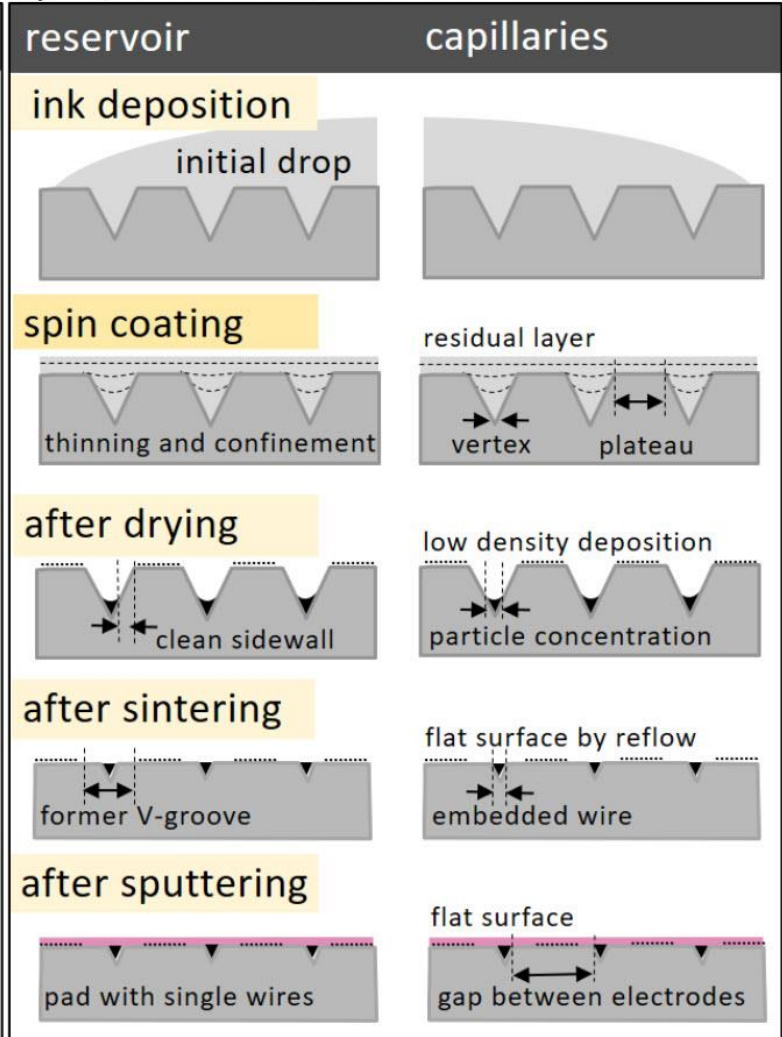

Figure 4. Concept of filling of V-shaped grooves by a) capillary filling from reservoirs to supply the empty grooves, and by b) spin coating. Here, reservoirs are structured with the same size and shape as the capillaries. While for capillary filling convective flow of ink from the reservoir enables the continuous transport of nanoparticles along capillaries during evaporation of the solvent, for spin coating the amount of ink nanoparticles settling down in the vertex of the $\mathrm{V}$-grooves is mostly defined by the dilution of the dispersion.

Figure 4 shows the process steps of capillary filling and spin coating on the same patterned structure. The first major difference between spin coated and capillary-filled wires is that for capillary spreading the sidewalls of the V-grooves are decorated with nanoparticles, but for spin coating the nanoparticles only deposit at the bottom and do not stick to the sidewalls. As seen in the schematic and the cross sectional SEM image of Figure 4a and Figure 7a-b, in case of capillary filling, the Ag nanoparticles deposit on the entire surface of the sidewalls after the spreading of the ink and evaporation of the solvent. This is because during evaporation of the solvent more liquid ink is transported continuously along the capillary from the reservoirs [16]. Since the channel is filled with ink up to the rim, more nanoparticles are transported into the channel while a significant amount of solvent is evaporated. This is in contrast to the spin coating method (Figure 5), where a collection of nanoparticles deposits in the bottom of the Vgroove, thus enabling a linewidth reduction [22]. This confinement develops because the inks are further diluted with IPA, which results in more than a factor of 8 times more solid content of the ink used for capillary filling than for spin coating. For capillary filling, the thickness of the Ag wire on the top region of the sidewalls is limited to around 100-200 nm. However, it still fully functions as an electrode to transport electrons from the overlaying $\mathrm{ZnO}$ layer. The middle part of the wire is $1-2 \mu \mathrm{m}$ thick, depending on the region of the IDEA. The wires of the 
spin coated IDEAs are homogeneously $800 \mathrm{~nm}$ wide after sintering and their cross section shows a triangular shape.

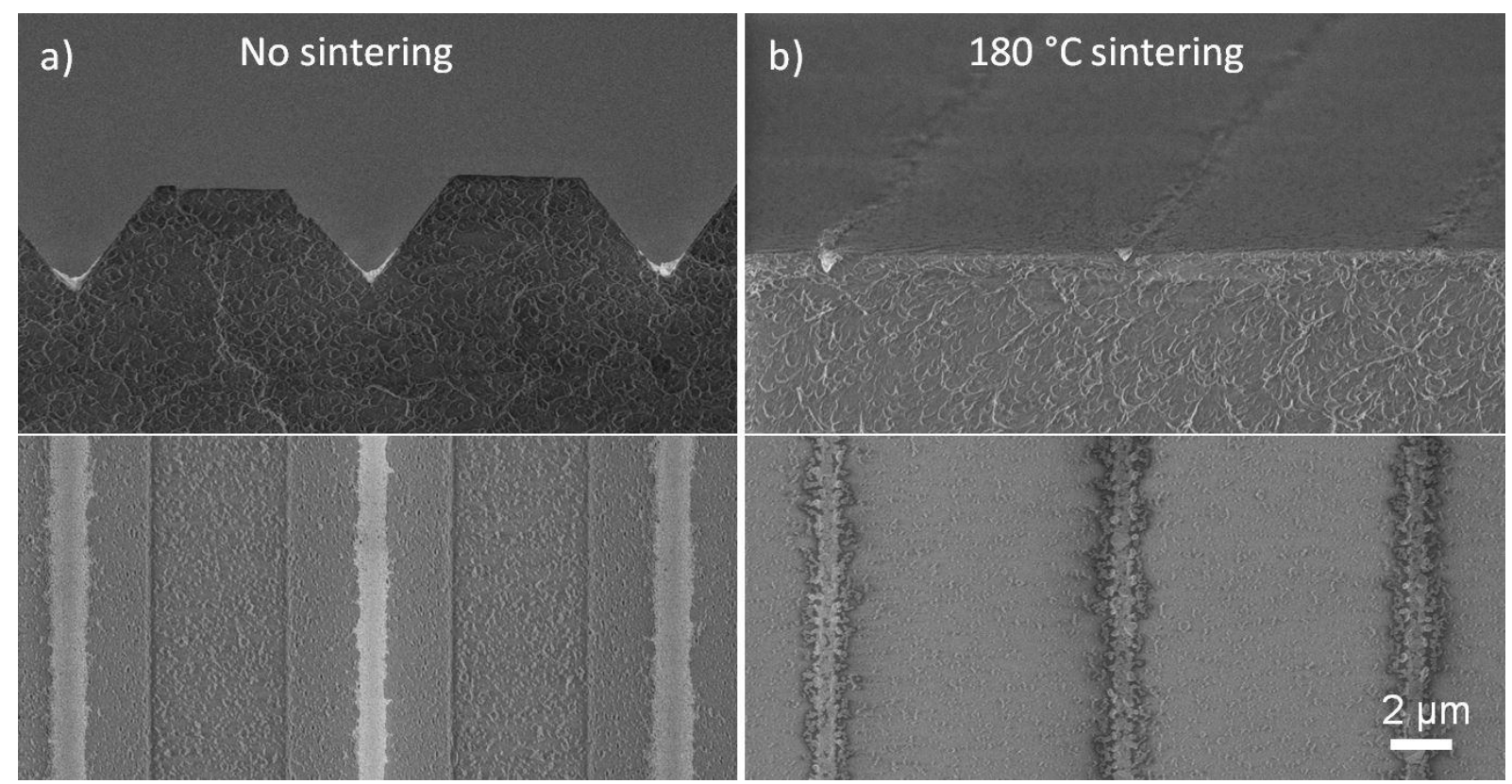

Figure 5. Cross sectional (top) and top view (bottom) SEM micrographs of spin coated Ag wires a) before and b) after sintering. The ink nanoparticles collect in the vertex during spin coating and the PMMA flattens during the $180{ }^{\circ} \mathrm{C}$ sintering.

The second difference between the two processing methods is the behavior of the PMMA samples after sintering. For the spin coating case, the sidewalls of the V-grooves made of PMMA are smoothened out by thermal reflow in the areas without nanoparticle coverage due to surface energy minimization (Figures $4 b$ and 5a-b). The PMMA substrate softens during the sintering of the ink and transforms its surface shape causing the ridges to sink and the Vgrooves to become initially rounded and then completely flattened. This flattening property can be tuned by the sintering temperature or by modifying the $\mathrm{T}_{\mathrm{g}}$ of the polymeric material. While $150{ }^{\circ} \mathrm{C}$ is adequate for sintering the Ag nanoparticles according to the manufacturer's data sheets, heating to $180{ }^{\circ} \mathrm{C}$ is necessary for complete flattening of the topography of the PMMA film. Figure 6 shows the flattening effect of different temperatures on the PMMA film after spin coating diluted Ag ink; it can be seen that complete flattening of the $150 \mu \mathrm{m}$ thick PMMA film occurs at $180{ }^{\circ} \mathrm{C}$. During sintering, the nanoparticles sinter together and a thin silver wire forms in a small $\mathrm{V}$-groove surrounded by a flattened-out surface. The sintered wire keeps the initial V-shape after sintering, however it is slightly flattened compared to the initial $54.74^{\circ}$ angle [22]. Since the final metal structure is initially on the bottom of the polymer grooves, the addition of further layers would be difficult in a roll-to-plate process line, e.g. by gravure printing. Therefore, the removal of the $\mathrm{V}$-groove topography is advantageous in case further process steps are planned on the sample after wire fabrication. 

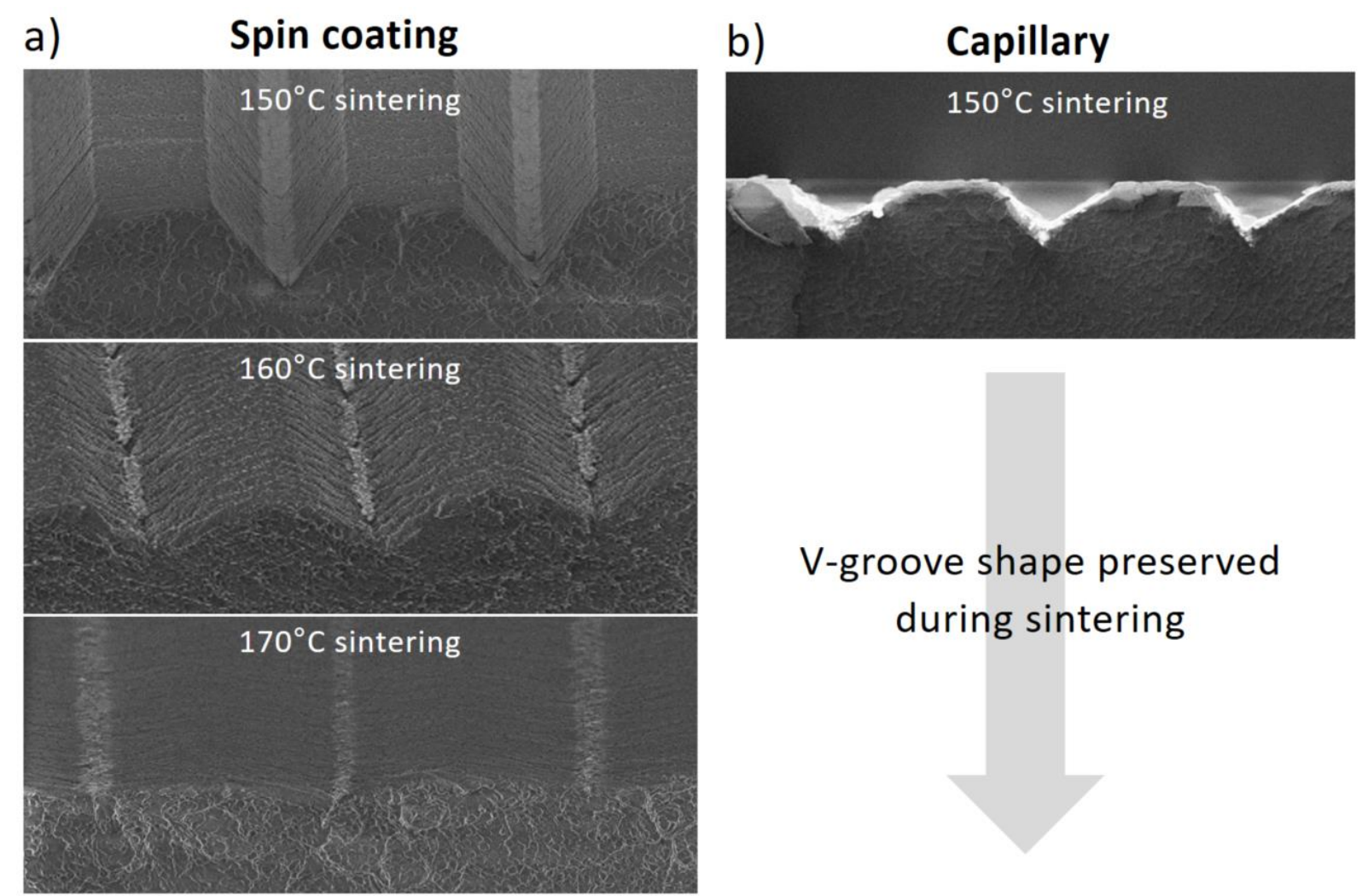

during sintering
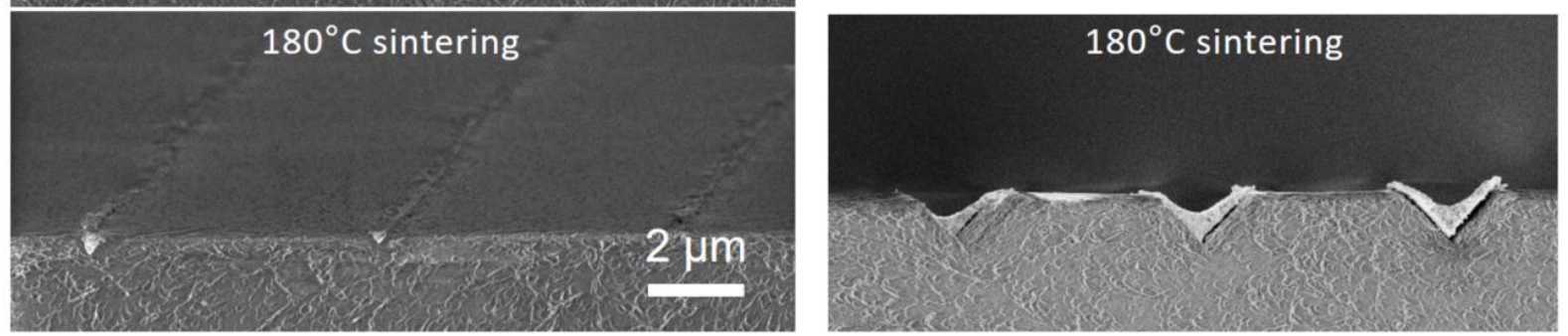

Figure 6. Effect if different sintering temperatures on the PMMA film. a) Flattening of the Vgrooves due to thermal reflow after spin coating Ag nanoparticle ink diluted with 1:59 IPA. b) Inhibited topography flattening due to the mechanically stabilizing Ag layer in the capillary filled V-grooves.

Capillary filled samples, on the other hand, do not experience such shape transformation since the entire $\mathrm{V}$-groove is covered with a closed film of nanoparticles after the spreading of the Ag ink (Figure 7a). The deposited nanoparticles transform into an Ag layer during sintering which inhibit the flattening of the $\mathrm{V}$-grooves at $150{ }^{\circ} \mathrm{C}$ sintering temperature (Figure $7 \mathrm{~b}$ ). Only a slight reduction is observed in the angle of the groove. Samples were also tested after sintering at $180^{\circ} \mathrm{C}$, and the $\mathrm{V}$-grooves were preserved by the $\mathrm{Ag}$ layer similarly to when sintering at 150 ${ }^{\circ} \mathrm{C}$ (Figure 6b). In contrast to the flattening observed for spin coated samples, the preserved surface topography would enable subsequent filling with another material. 


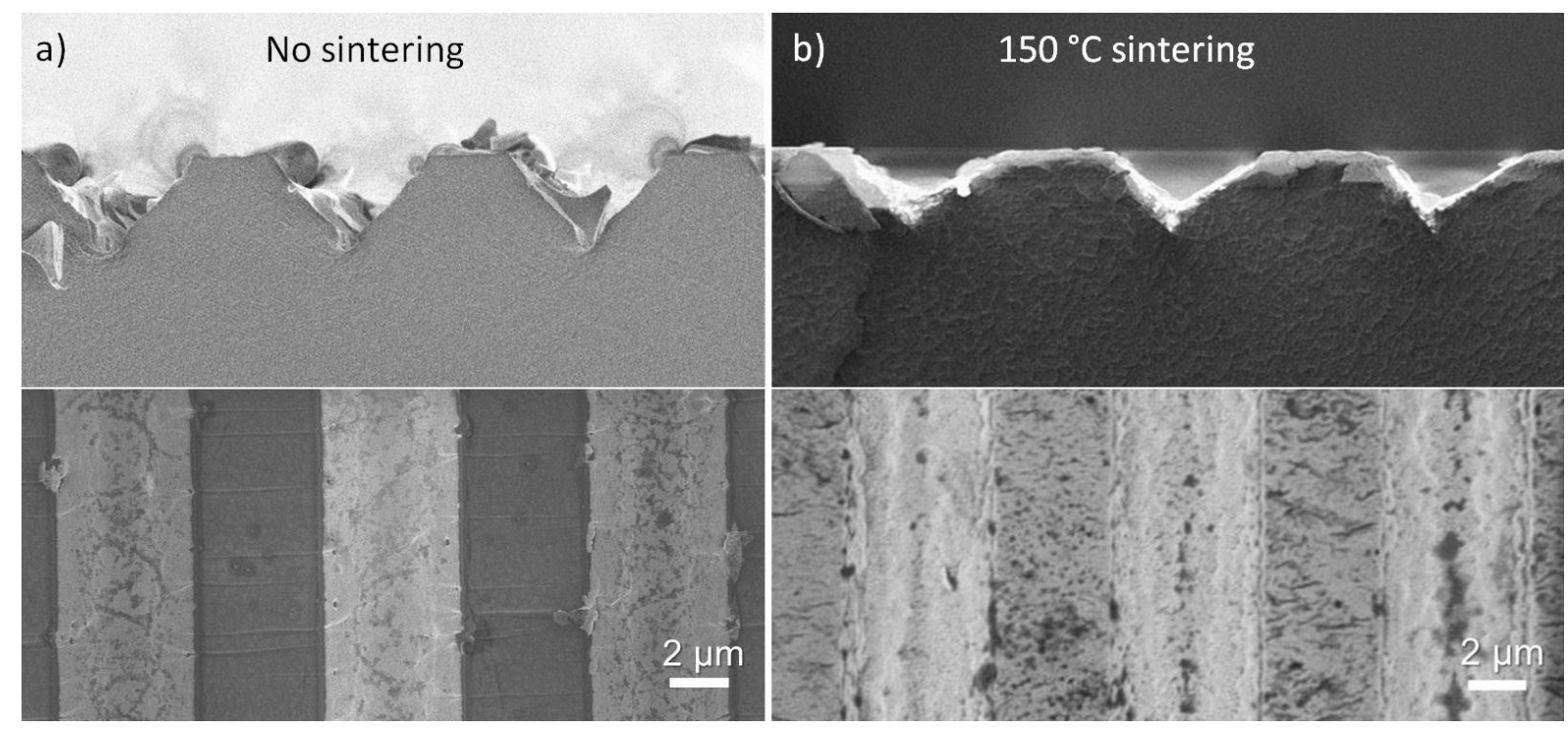

Figure 7. Cross sectional (top) and top view (bottom) SEM micrographs of capillary filled Ag wires a) before and b) after sintering. The ink nanoparticles cover the sidewalls of the trenches, which prohibits the flattening of the PMMA substrate during the $150{ }^{\circ} \mathrm{C}$ sintering. The artifacts on the top of the trenches on the before sintering cross section image originate from the charging of the sample during SEM.

\subsection{UV-sensors with interdigitated electrodes}

\section{Design consideration for interdigitated electrode arrays for $U V$-sensors}

An IDEA is ideal for the harvesting of the photoelectrons generated by UV-illumination that results in a photocurrent, since - when coated with a semiconductor - it enables to place electrodes near to the location where the photons generate positive and negative charges. In the research presented here, the IDEAs were coated with a $70 \mathrm{~nm}$ thin layer of $\mathrm{ZnO}$, a semiconductor with a bandgap in the range of $3.3 \mathrm{eV}$ (equivalent to a wavelength of $376 \mathrm{~nm}$ ). Therefore, the $\mathrm{ZnO}$ sensor device acts as a photoconductor sensitive only in the UV region. For the shape of the electrodes, V-groove capillaries have been selected, since its geometry avoids problems that would occur in U-grooves, such as thinning of the $\mathrm{ZnO}$ layer due to shadowing and inhomogeneous coating of vertical sidewalls during sputtering. The thickness of the Ag wires does not play a significant role because their resistance in the range of $100 \Omega$ is by magnitudes lower than the deposited $\mathrm{ZnO}$ (in the range of $1 \mathrm{G} \Omega$ between two wires).

\section{Measurement setup}

The polymer films with the IDEAs were examined in a simple setup with an LED placed $10 \mathrm{~mm}$ above the IDEA structure. The setup of the measurement can be seen in Figure 8a. The devices were measured at room temperature in air when irradiated with $355 \mathrm{~nm}$ and $385 \mathrm{~nm}$ UV light and without irradiation in the dark. $385 \mathrm{~nm}$ light is in the upper range of the UV spectrum. The I-V characteristics of one specific $\mathrm{ZnO}$ sensor are shown in Figure $8 \mathrm{~b}$. When irradiating with $355 \mathrm{~nm}$ UV light, high currents were measured (140 nA at $5 \mathrm{~V}$ ). On the other hand, only very low photocurrent $(11 \mathrm{nA}$ at $5 \mathrm{~V})$ was observed when irradiating the sensor with $385 \mathrm{~nm}$. This can be explained with the bandgap characteristics of $\mathrm{ZnO}$ that absorbs light below $376 \mathrm{~nm}$ but is almost transparent at $385 \mathrm{~nm}$. Since absorption of $\mathrm{ZnO}$ already starts at $3.2 \mathrm{eV}$ 
(equivalent to $388 \mathrm{~nm}$ ), and the LED spectral bandwidth is specified with $10 \mathrm{~nm}$, small absorption at $385 \mathrm{~nm}$ can be observed [28]. The dark current was zero when no illumination occurred. The I-V characteristics of the sensor device in all three situations are close to linear.
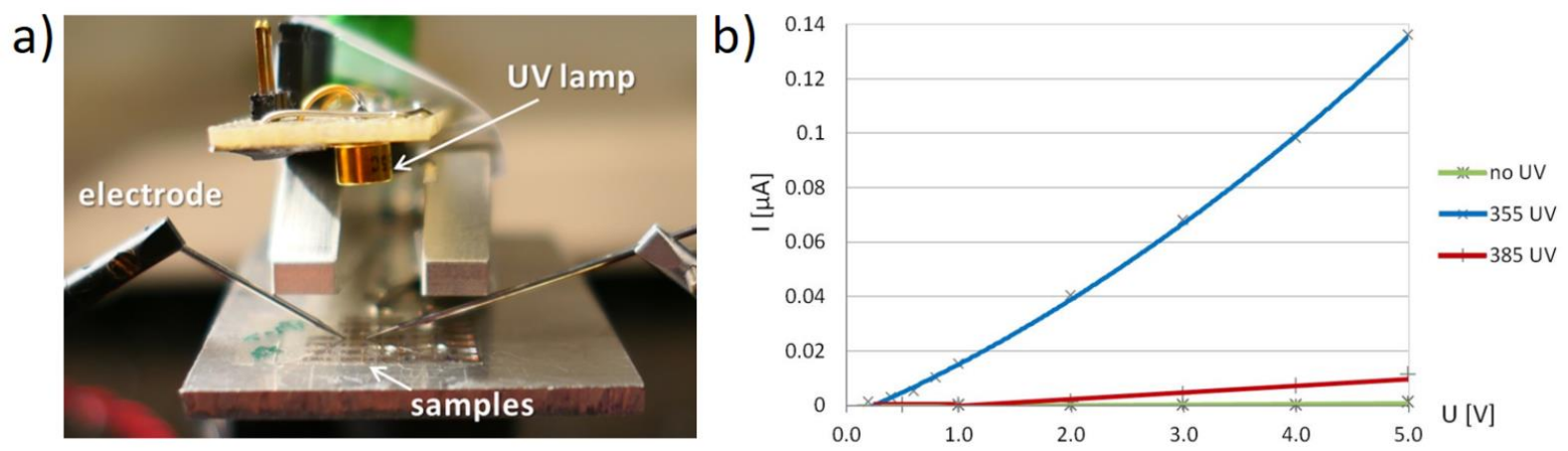

Figure 8. a) Photograph of the measurement setup with a film covered with IDEAs. UV lamp distance: $10 \mathrm{~mm}$. b) I-V curve measured in dark and in UV irradiation of 355 and $385 \mathrm{~nm}$ on a film made by capillary spreading.

The resistivity of the $\mathrm{ZnO}$ films during $355 \mathrm{~nm}$ UV illumination was calculated from the average current flow of $0.21 \mu \mathrm{A}$ measured at $5 \mathrm{~V}$ on $(5 / 10)$ samples. Based on the fact that 50 electrodes on the each side are interdigitated in the structure, 99 electrically parallel $\mathrm{ZnO}$ sections can be considered. This fact, plus the geometrical properties of each $\mathrm{ZnO}$ piece $(1 \mathrm{~mm}$ wide, $4.6 \mu \mathrm{m}$ long, $70 \mathrm{~nm}$ thick) gives us a resistivity of the deposited $\mathrm{ZnO}$ film to be $3.59 \times$ $10^{6} \Omega \mathrm{cm}$. Since the resistivity of the Ag wires are in the range of $10^{-6} \Omega \mathrm{cm}$ [22], they were not considered in the calculation. Figure 9a shows the response/recovery curve of the $\mathrm{ZnO}$ sensor when switching on and off the $355 \mathrm{~nm}$ UV light and the voltage source. An exponential growth and decay trend $[29,30]$ can be seen which takes place in the first few minutes after switching. This can be explained by the photo-generation of electron-hole pairs with light illumination that quickly recombine after switching off the UV light. On top of that, oxygen and water adsorption on the film surface has an additional role resulting in the extraction of free electrons from $\mathrm{ZnO}$ [31]. Our measurements show that the $\mathrm{ZnO}$ sensor reacts well to continuous on and off switching.

Figure $9 \mathrm{~b}$ shows a comparison between capillary filling and spin coating. In general, capillary filled samples exhibit a higher photocurrent than spin coated samples. Since the distance of the $\mathrm{ZnO}$ layer is wider at the spin coated arrangement, the (5/10) arrangement has distances of 4.6 vs. $9.6 \mu \mathrm{m}$ for the spin coated and capillary filled samples, respectively. The topography of the two process methods and the shape of the Ag wire does not have a role in the electrical measurements due to its high conductivity $\left(4.6 \times 10^{6} \Omega \mathrm{m}\right)$ [22]. The $\mathrm{ZnO}$ layer between two Ag wires is always lying on a flat surface, either 1) in case of capillary: between two grooves or 2) for spin coating: on the flattened PMMA surface. Therefore, the only difference between the two techniques results in the distance of the Ag wires. The photocurrents measured for capillary filled sensors, however, were only 1.2-1.5 times higher than the expected 2 times we would expect from the wire distances. We generally observed that spin coating resulted in homogeneous faultless wires without any interruptions, while capillary filling often suffered from faulty electrodes with no sensing capabilities whatsoever. This is probably the result of the more controlled evaporation of the solvent in case of spin coating, which occurs all over the surface without significant flow of ink along the capillaries. Figure 9c shows the characteristics differences of capillary filled sensors due to the differences of the distances of 
the electrodes. At shorter distances (5/10), the current is close to twice as high as for (5/15) and about 5 times higher than for $(5 / 20)$. This shows underachievement for the $(5 / 20)$ compared to the expected 3 times value.

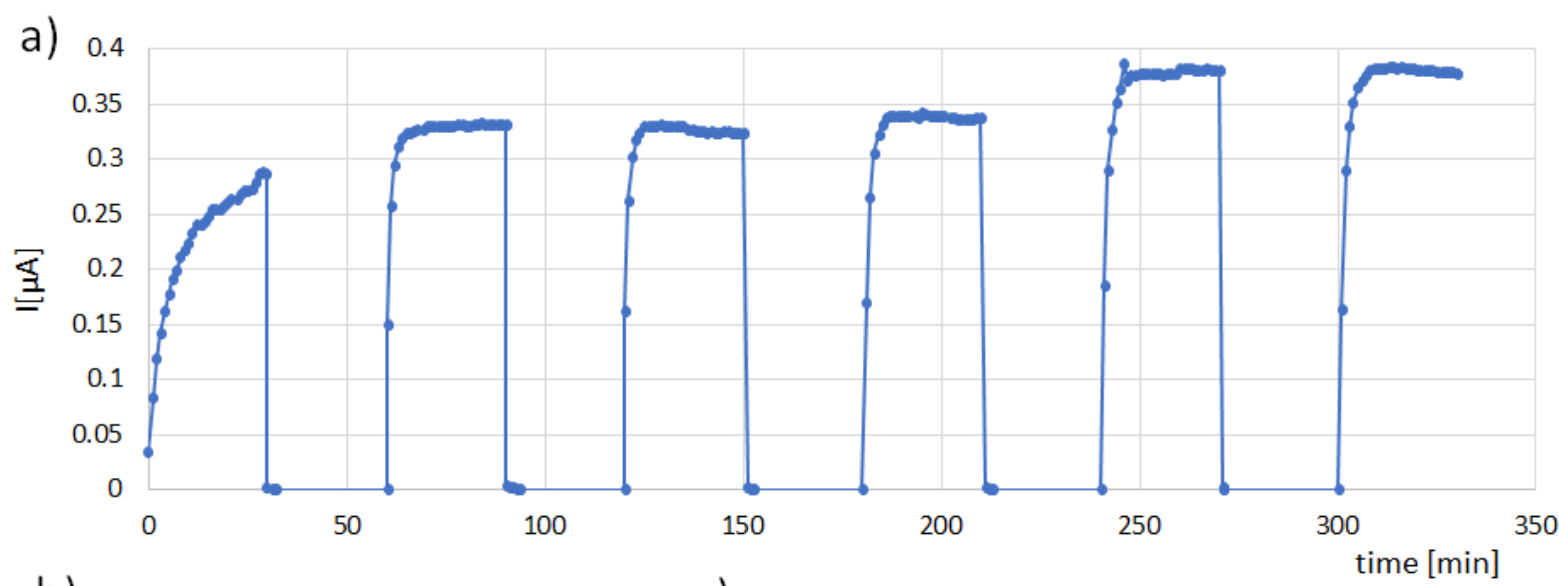

b)

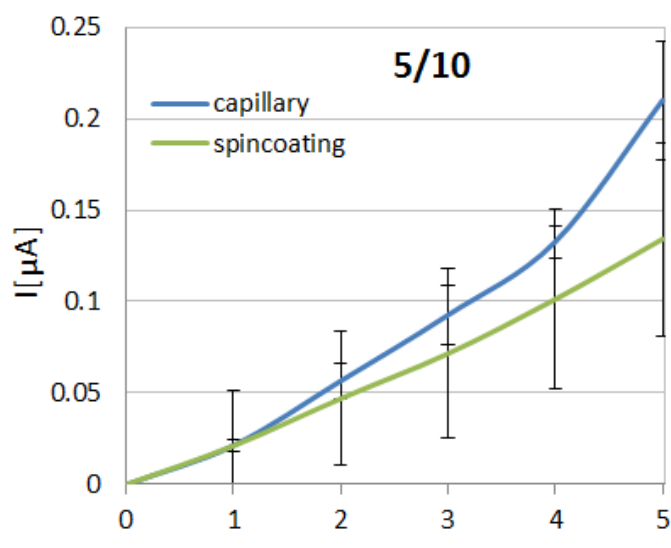

c)

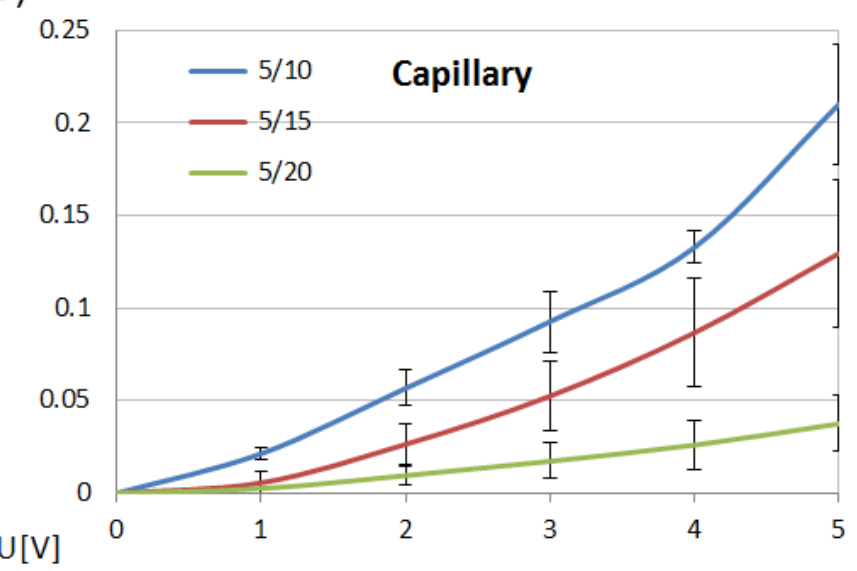

Figure 9. IDEA properties measured at $355 \mathrm{~nm}$ UV irradiation; a) Graph of the response/recovery curve. b) I-V curve comparison between capillary filling and spin coating for $(5 / 10)$. c) I-V curve comparison of different distances of $\mathrm{V}$-grooves for capillary filling with different distances $4.6 \mu \mathrm{m}, 9.6 \mu \mathrm{m}$ and $14.6 \mu \mathrm{m}$, here denoted with $(5 / 10),(5 / 15)$ and $(5 / 20)$.

\section{Conclusion}

The fabrication of an UV-sensor with IDEA configuration demonstrates the capability of self-confinement by capillary effects, and that open microfluidics is a viable approach to use low-resolution additive techniques in combination with pre-patterned substrates for capillary networks. The advantage of spin coating is the generation of wires with smaller widths, high yield and the ability to flatten the substrate due to the confinement in the vertex. Similarly to the original SCALE concept, capillary filling is more flexible, enables deposition by drop-on demand, and would qualify for large area substrates as used in printed electronics. Currently, sputtering has been used to cover the Ag wire network with $\mathrm{ZnO}$. The thickness was thin enough that the wire geometry could still be analyzed for visible defects after measurement. However, for low cost fabrication, it is known that sol-gel processes can be used which allow the use of spray coating. Nevertheless, it could be envisaged that the same processes, i.e. pre-patterning 
of a polymeric surface with capillaries, can be used with other replication processes such as injection molding. Then inks can be applied and by adding coatings, sensors integrated into the surface of a polymeric part. Furthermore NIL is upscalable to large area and high throughput by using roll-to-plate (R2P) or roll-to-roll (R2R) techniques [32]. Here, automated inking techniques would allow for filling capillaries in a drop-on-the-spot approach.

Acknowledgements: The authors are grateful to Konrad Vogelsang in PSI for his work in nanoimprint replication, Dario Marty for stamp fabrication, Barbora Křivová and Sijia Xie for SEM images. At EMPA we thank Galo Torres Sevilla for sputtering of $\mathrm{ZnO}$ and Sami Bolat, Yaroslav Romanyuk and Jakob Heier for their support. The financial support from project FOXIP [33] in the framework of the Strategic Focus Area (SFA) Advanced Manufacturing of the ETH Board is acknowledged.

Patent information: European Patent Application No. 18173943.4-1022, filed on 24 May 2018.

\section{References}

[1] Gao M, Li L and Song Y 2017 Inkjet printing wearable electronic devices Journal of Materials Chemistry C 5 2971-93

[2] Grau G, Cen J, Kang H, Kitsomboonloha R, Scheideler W J and Subramanian V 2016 Gravureprinted electronics: recent progress in tooling development, understanding of printing physics, and realization of printed devices Flexible and Printed Electronics 1023002

[3] Hu Q, Wu H, Sun J, Yan D, Gao Y and Yang J 2016 Large-area perovskite nanowire arrays fabricated by large-scale roll-to-roll micro-gravure printing and doctor blading Nanoscale 8 5350-7

[4] Fukuda K and Someya T 2017 Recent Progress in the Development of Printed Thin-Film Transistors and Circuits with High-Resolution Printing Technology Advanced Materials 29 1602736

[5] Sung J, Kang B J and Oh J H 2013 Fabrication of high-resolution conductive lines by combining inkjet printing with soft lithography Microelectronic Engineering 110 219-23

[6] Schift H, Jaszewski R W, David C and Gobrecht J 1999 Nanostructuring of polymers and fabrication of interdigitated electrodes by hot embossing lithography Microelectronic Engineering 46 121-4

[7] Schift H 2008 Nanoimprint lithography: An old story in modern times? A review Journal of Vacuum Science \& Technology B: Microelectronics and Nanometer Structures Processing, Measurement, and Phenomena 26 458-80

[8] Xia D, Johnson L M and López G P 2012 Anisotropic Wetting Surfaces with One-Dimesional and Directional Structures: Fabrication Approaches, Wetting Properties and Potential Applications Advanced Materials 24 1287-302

[9] Romero L A and Yost F G 2006 Flow in an open channel capillary Journal of Fluid Mechanics 322 109-29

[10] Olanrewaju A, Beaugrand M, Yafia M and Juncker D 2018 Capillary microfluidics in microchannels: from microfluidic networks to capillaric circuits Lab on a Chip 18 2323-47

[11] Hyun W J, Secor E B, Zare Bidoky F, Walker S B, Lewis J A, Hersam M C, Francis L F and Frisbie C D 2018 Self-aligned capillarity-assisted printing of top-gate thin-film transistors on plastic Flexible and Printed Electronics 3035004

[12] Mahajan A, Hyun W J, Walker S B, Rojas G A, Choi J-H, Lewis J A, Francis L F and Frisbie C D 2015 A Self-Aligned Strategy for Printed Electronics: Exploiting Capillary Flow on Microstructured Plastic Surfaces Advanced Electronic Materials 11500137 
[13] Hyun W J, Bidoky F Z, Walker S B, Lewis J A, Francis L F and Frisbie C D 2016 Printed, SelfAligned Side-Gate Organic Transistors with a Sub-5 $\mu \mathrm{m}$ Gate-Channel Distance on Imprinted Plastic Substrates Advanced Electronic Materials 21600293

[14] Hyun W J, Kumar S, Francis L F and Frisbie C D 2018 Open-channel microfluidic diodes based on two-tier junctions Applied Physics Letters 113193701

[15] Gravesen P, Branebjerg J and Jensen O S 1993 Microfluidics-a review Journal of Micromechanics and Microengineering 3 168-82

[16] Horváth B, Křivová B and Schift H 2019 Nanoimprint meets microfluidics: Development of metal wires from nanoparticle ink filled capillaries Micro and Nano Engineering 3 22-30

[17] Lone S, Zhang J M, Vakarelski I U, Li E Q and Thoroddsen S T 2017 Evaporative Lithography in Open Microfluidic Channel Networks Langmuir 33 2861-71

[18] Washburn E W 1921 The Dynamics of Capillary Flow Physical Review 17 273-83

[19] Yang D, Krasowska M, Priest C, Popescu M N and Ralston J 2011 Dynamics of Capillary-Driven Flow in Open Microchannels The Journal of Physical Chemistry C 115 18761-9

[20] Chen T 2015 Capillary Force-Driven Fluid Flow in Open Grooves with Different Sizes Journal of Thermophysics and Heat Transfer 29 594-601

[21] Rye R R, Yost F G and O'Toole E J 1998 Capillary Flow in Irregular Surface Grooves Langmuir 14 3937-43

[22] Horváth B, Křivová B, Bolat S and Schift H 2019 Fabrication of Large Area Sub-200 nm Conducting Electrode Arrays by Self-Confinement of Spincoated Metal Nanoparticle Inks Advanced Materials Technologies 41800652

[23] Ali G M and Chakrabarti P 2010 ZnO-based interdigitated MSM and MISIM ultraviolet photodetectors Journal of Physics D: Applied Physics 43415103

[24] Rao V K, Abhinav K V, Karthik P S and Prakash S S 2015 Conductive silver inks and their applications in printed and flexible electronics RSC Advances 5 77760-90

[25] Tie L, Guo Z and Liu W 2015 Anisotropic wetting properties on various shape of parallel grooved microstructure Journal of Colloid and Interface Science 453 142-50

[26] den Otter M W 2002 Approximate expressions for the capacitance and electrostatic potential of interdigitated electrodes Sensors and Actuators A: Physical 96 140-4

[27] Le D D, Nguyen T N N, Doan D C T, Dang T M D and Dang M C 2016 Fabrication of interdigitated electrodes by inkjet printing technology for apllication in ammonia sensing Advances in Natural Sciences: Nanoscience and Nanotechnology 7025002

[28] Srikant V and Clarke D R 1998 On the optical band gap of zinc oxide Journal of Applied Physics 83 5447-51

[29] Law J B K and Thong J T L 2006 Simple fabrication of a ZnO nanowire photodetector with a fast photoresponse time Applied Physics Letters 88133114

[30] Ahn S E, Lee J S, Kim H, Kim S, Kang B H, Kim K H and Kim G T 2004 Photoresponse of sol-gelsynthesized ZnO nanorods Applied Physics Letters 84 5022-4

[31] Panda S K and Jacob C 2012 Preparation of transparent ZnO thin films and their application in UV sensor devices Solid-State Electronics 73 44-50

[32] Ahn S H and Guo L J 2009 Large-Area Roll-to-Roll and Roll-to-Plate Nanoimprint Lithography: A Step toward High-Throughput Application of Continuous Nanoimprinting ACS Nano 3 230410

[33] FOXIP Project - Functional OXIdes Printed on Polymers and Paper, Strategic Focus Area (SFA) Advanced Manufacturing) 